\title{
Comparison of Different Methods of Abutment Splinting and Attachments on Teeth Kinetics (Part I)
}

\author{
${ }^{1}$ Ahmed N Elsherbini, ${ }^{2}$ Wilhelm Niedermeier
}

\begin{abstract}
Aim: The aim of this study was to measure the effect of different attachments: telescopic crown, double Akers' (DA) clasp, distal clasp (DC)-retained removable partial dentures (RPDs), and cantilever bridge on the intrusive movement of the abutment teeth.
\end{abstract}

Materials and methods: A model imitating mandibular ridge of Kennedy Class I was fabricated with first and second premolar as abutments. A telescopic crown, DA, DC-retained RPDs, and cantilever bridge were fabricated. Each partial denture was placed at a time on the model; $50 \mathrm{~N}$ force was applied on each partial denture using ZWICK universal testing machine. The intrusion of the abutments was recorded using SOLARTON device. Data were collected and tabulated.

Results: The mean values of the intrusive movement in telescopic separated (TS) RPD, telescopic joined (TJ) RPD, DA RPD, DC RPD, and cantilever bridge in tooth 35 were 147, 75, 57,334 , and $307 \mu \mathrm{m}$ respectively, and in tooth 45 were 136 , $81,65,435$, and $335 \mu \mathrm{m}$ respectively.

Conclusion: It can be concluded from the retrieved data that DA clasp-retained RPD caused less intrusion of the abutments than telescopic crown-retained RPD, followed by the DC than cantilever bridge.

Clinical significance: The DA clasp is most favorable in the utilization in mandibular Kennedy Class I situation followed by telescopic crowns.

Keywords: Abutment intrusion, Cantilever bridge, Double Akers, Telescopic crown.

How to cite this article: Elsherbini AN, Niedermeier W. Comparison of Different Methods of Abutment Splinting and Attachments on Teeth Kinetics (Part I). World J Dent 2017;8(5):352-357.

Source of support: Nil

Conflict of interest: None

\section{INTRODUCTION}

Removable partial dentures are necessary to provide restoration of the mandible and the maxilla in cases of a shortened dental arch. There are several classes of remaining dentitions, whereby the posterior distal

\footnotetext{
${ }^{1}$ Department of Prosthodontics, Modern Sciences and Arts University, Cairo, Egypt

${ }^{2}$ Department of Prosthodontics, University of Cologne, Cologne Germany

Corresponding Author: Ahmed N Elsherbini, Department of Prosthodontics, Modern Sciences and Arts University, Cairo Egypt, e-mail: ahmed-elsherbini@live.com
}

extension is the most frequent as a result of the early loss of molars. In this class with remaining anterior teeth (Kennedy Class I), commonly a bilateral distal extension RPD is applied to reconstruct the lost teeth. ${ }^{1,2}$

The denture is teeth supported anteriorly, while it is tissue supported posteriorly. This difference in support between the anterior and posterior ends of these bases results in undesirable movements that cause damage to the supporting tissues (both abutment teeth and residual ridges) due to the difference of tissue resiliency.

Many treatment modalities have been suggested to overcome related problems of this class of edentulous ridges.

Cantilever bridges deliver good masticatory functions and improved the supporting structures by providing good occlusal and neuromuscular stability. ${ }^{3}$ However, it was also proved that cantilever bridges induced tilting and rotational movements on the abutments rather than along vertical long axis of the abutment. Telescopic crowns were also introduced as a treatment option, where it equalized forces on the supporting structures and decreased the tilting of the abutments. ${ }^{4}$

Another suggestion was the DA clasp (Bonwill), with placement of the support away from the distal extension and engaging distobuccal undercut instead of the mesiobuccal undercut. It remarkably reduced the transmitted forces to the terminal abutments and improved the rate of residual bone resorption. ${ }^{5,6}$

The location of loading points influenced both the magnitude and direction of the abutment tooth and denture base movement. ${ }^{7}$

There was a need to carry out a research that highlights the best treatment options for such situation, so the aim of this study is to compare the cantilever bridge, telescopic crowns, DA clasp, and DC on the intrusion of the abutments in Kennedy Class II situation.

\section{Hypothesis}

There is no effect on the intrusion of abutments when using different attachments.

\section{MATERIALS AND METHODS}

Due to many variables in the biomechanics of different treatment options, and the ethical problems of in vivo research, our study was limited to a pseudorealistic in 


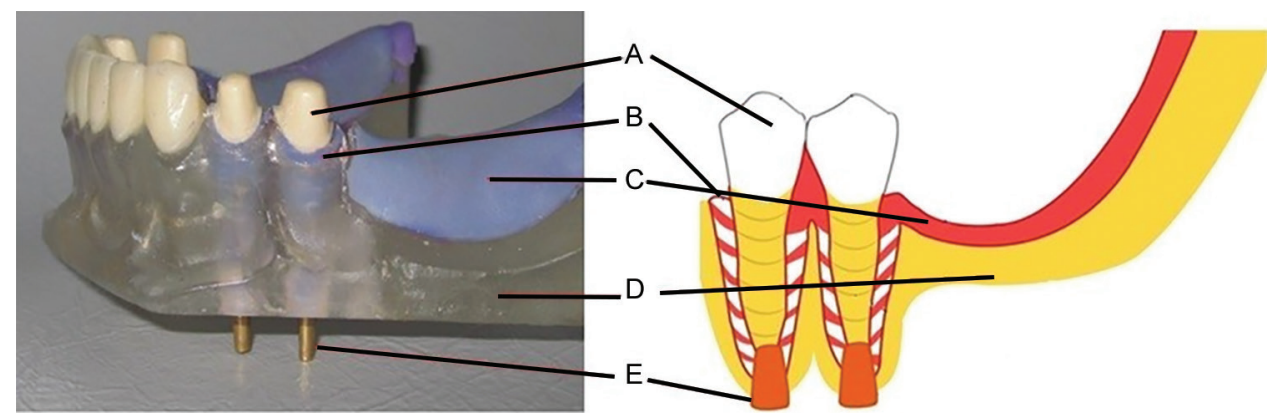

Fig. 1: Fabricated model with bilateral distal extension: (A) Abutment teeth FRASACO ${ }^{\circledR}$; (B) silicon SILASOFT N ${ }^{\circledR}$ around abutments; (C) silicon SILASOFT $\mathrm{N}^{\circledR}$ on saddle; (D) acrylic PALAPRESS ${ }^{\circledR}$ VARIO; and (E) dowel pin

vitro model, which bears a resemblance to the natural tooth abutment. A model with bilateral distal extension was fabricated. The first and second premolars were chosen as abutments, and dowel pins were extended from the abutments for measuring procedure (Fig. 1).

\section{Fabrication of Treatment Options}

\section{Cantilever Bridge}

A replica mold of the model was made and poured with high-strength HERAEUS ${ }^{\circledR}$ dental stone. Wax pattern was made on the prepared abutments using inlay wax S-U-ÄSTHETIKWACHS-O ${ }^{\circledR}$; the extension pontic was made with inlay wax. The wax pattern was tried-in first on the original model, and then invested with $\mathrm{BEGO}^{\circledR}$ investment material, casted with BEGO WIROBOND ${ }^{\circledR}$ chromium cobalt alloy, finished, and polished.

\section{Telescopic Crown}

Replica mold was poured with high-strength HERAEUS ${ }^{\circledR}$ dental stone. Wax patterns of the primary crowns were milled using DEGUSSA ${ }^{\circledR}$ milling machine, using cylindrical burs to produce a parallel pattern. The primary crowns were joined as one unit. Wax pattern was invested using $\mathrm{BEGO}^{\circledR}$ investment material. The casted primary crowns were finished using DEGUSSA ${ }^{\circledR}$ milling machine, to produce parallel walled primary crowns. Wax patterns for the secondary crowns were made. Wax pattern was invested with $\mathrm{BEGO}^{\circledR}$ investment material, then finished and polished. A replica mold of the model with the primary and secondary crowns was made. Wax pattern of the metal framework was done, invested, finished, and polished. The metal framework and the secondary crowns were welded together by laser by Dental Technik HARTWICH. Acrylic teeth made from PALAVIT ${ }^{\circledR}$ acrylic and acrylic for the freeend saddle made from PALAPRESS ${ }^{\circledR}$ was used.

\section{Conventional Clasp (DA Clasp and DC)}

Replica mold of the model was poured with high-strength HERAEUS $^{\circledR}$ dental stone. Wax pattern was made on the prepared abutments using blue inlay wax; occlusal rests were prepared on the distal and mesial thirds of the first and second premolars respectively, and on the occlusodistal third in the second premolar. Investment using $\mathrm{BEGO}^{\circledR}$ investment materials, casted with BEGOWIROBOND ${ }^{\circledR}$ chromium cobalt alloy, was finished and polished. The crowns were cemented to the cast using temporary cement Temp Bond ${ }^{\circledR}$ (Kerr Company) and a replica mold was made using additional silicon and poured with investment material. Wax pattern of the partial denture was made with ready-made wax, then invested, casted, and finished. Metal framework was tried-in on the model with the crowns retained in place with temporary cement. The acrylic teeth were added to the framework, and acrylic resin PALAPRESS ${ }^{\circledR}$ was used. Two designs of RPD were constructed; the DA clasp (Bonwill) and the DC.

\section{Measuring Procedure}

The model was fixed with a screw to a holding rod, which in turn was fixed with a screw to the ZWICK machine, and then the load applicator of the ZWICK machine was centralized on the model. The SOLARTON sensor was lined up parallel and on the same line with abutment to contact the end of the dowel pin of each abutment, and the sensor was held in place using special arm device fixed to the holding rod. A metallic rod was placed on the model with the different prostheses, and the load applicator of the ZWICK machine applied the force at the center of this metallic rod. A special plastic ring was made, attached on the prostheses at the indicated positions, and numbered (Fig. 2).

\section{Steps of Measuring}

The prostheses were placed in position on the testing model.

For the TJ, TS, DA clasp and DC RPD, three positions were tested:

1. Position one: front of the saddle, 


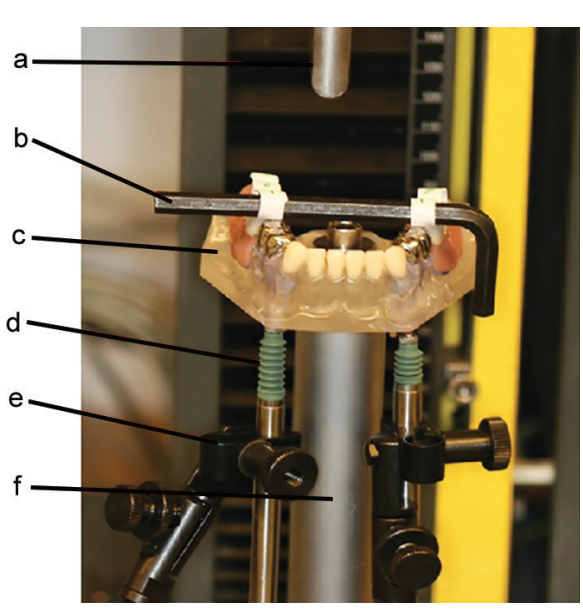

Fig. 2: Model was fixed with a screw to a holding rod. (a) Loading applicator, (b) metallic rod, (c) testing model, (d) Solarton sensor, (e) holding arm, (f) holding rod

\section{Position two: middle of the saddle,}

3. Position three: end of the saddle.

For the cantilever bridge, there was only one position on the pontic.

For the TJ, TS, DA, and cantilever bridge, the teeth 34, 35,44 , and 45 will be tested. For the DC only the teeth 35 and 45 were tested.

When the load with the ZWICK machine reached 50 $\mathrm{N}$, the sensor was started to take 20 readings at an interval of 1 second. Then, every 15 seconds a reading was taken until full recovery of the silicon, indicated by reaching the 0.000 mark.

\section{RESULTS}

\section{Force applied onto the Tooth-supported Region of the Saddle (Position 1)}

The mean values of the intrusive movement in TS RPD, TJ RPD, DA RPD, DC RPD, and cantilever bridge in tooth 35 were $147,75,57,334$, and $307 \mu \mathrm{m}$ respectively, and in tooth 45 were 136, 81, 65, 435, and $335 \mu \mathrm{m}$ respectively. Table 1 shows mean values of intrusion. The results of analysis of variance (ANOVA) revealed a statistically significant difference between the groups $(p<0.001)$. Bar chart shows mean values of intrusive movement for teeth 35 and $45(\mu \mathrm{m})$ for the different treatment options

Table 1: Mean values of intrusive movement of tooth $35(\mu \mathrm{m})$ for the different treatment options

\begin{tabular}{llllll}
\hline RPD type & Mean \pm SD & CV (\%) & Minimum & Maximum & Range \\
\hline TS RPD & $147 \pm 2.87$ & 1.76 & 142 & 150 & 8 \\
TJ RPD & $75 \pm 5.82$ & 8.38 & 69 & 89 & 20 \\
DA RPD & $57 \pm 2.6$ & 4.46 & 52 & 62 & 10 \\
DC retained & $334 \pm 19.1$ & 5.65 & 314 & 372 & 58 \\
Cantilever & $307 \pm 12.3$ & 3.95 & 297 & 333 & 36 \\
RPD & & & & &
\end{tabular}

SD: Standard deviation, CV: Coefficient of variation in an increasing order: TJ RPD; TS RPD; DA RPD; DCretained RPD; and (CA) cantilever bridge. Different capital letters denote a significant difference according to Tukey's pairwise comparison test for tooth 35, while different small letters denote a significant difference according to Tukey's pairwise comparison test for tooth 45. The mean values of the intrusive movement in TS RPD, TJ RPD, DA RPD, and cantilever bridge in tooth 34 were $139,55,58$, and $35 \mu \mathrm{m}$ respectively, and in tooth 44 were $135,46,61$, and $44.1 \mu \mathrm{m}$ respectively. The results of ANOVA revealed a statistically significant difference between the groups $(p<0.001)$. Bar chart shows mean values of intrusive movement for teeth 34 and $44(\mu \mathrm{m})$ for the different treatment options in an increasing order: TJ RPD, TS RPD, DA RPD, and (CA) cantilever bridge. Different capital letters denote a significant difference according to Tukey's pairwise comparison test for tooth 34, while different small letters denote a significant difference according to Tukey's pairwise comparison test for tooth 44 (Graph 1). In position 1, the abutment teeth were more active in supporting the RPDs, so the intrusion was higher. The intrusion of the abutment teeth decreased gradually with changing the positions, and moving toward the end of the distal extension base. This is because the residual ridge became more active in supporting the RPD, and larger portion of the acting load was carried out by the ridge, so lower loads were transmitted to the abutment teeth, resulting in lower intrusion in position 2 followed by position 3 .

\section{Force applied onto the Middle of the Saddle (Position 2)}

The mean values of the intrusive movement in TS RPD, TJ RPD, DA RPD, and DC RPD in tooth 35 were 89, 27.4, 35.3, and $118 \mu \mathrm{m}$ respectively, and in tooth 45 were 78.3, $37.2,43$, and $131.5 \mu \mathrm{m}$ respectively. The results of ANOVA revealed a statistically significant difference between the groups $(\mathrm{p}<0.001)$. Bar chart shows mean values of intrusive movement for teeth 35 and $45(\mu \mathrm{m})$ for the different treatment options in an increasing order: TJ RPD, TS RPD, DA RPD, and DC-retained RPD. Different capital letters denote a significant difference according to Tukey's pairwise comparison for the significant ANOVA for tooth 35 , while different small letters denote a significant difference according to Tukey's pairwise comparison test for tooth 45 . The mean values of the intrusive movement in TS RPD, TJ RPD, and DA RPD in tooth 34 were 83.3, 16.4, and $19.8 \mu \mathrm{m}$ respectively, and in tooth 34 were 72.1, 11.7, and $32.3 \mu \mathrm{m}$ respectively. The results of ANOVA revealed a statistically significant difference between the groups $(\mathrm{p}<0.001)$. Bar chart shows mean values of intrusive movement for teeth 34 and $44(\mu \mathrm{m})$ for the different treatment 

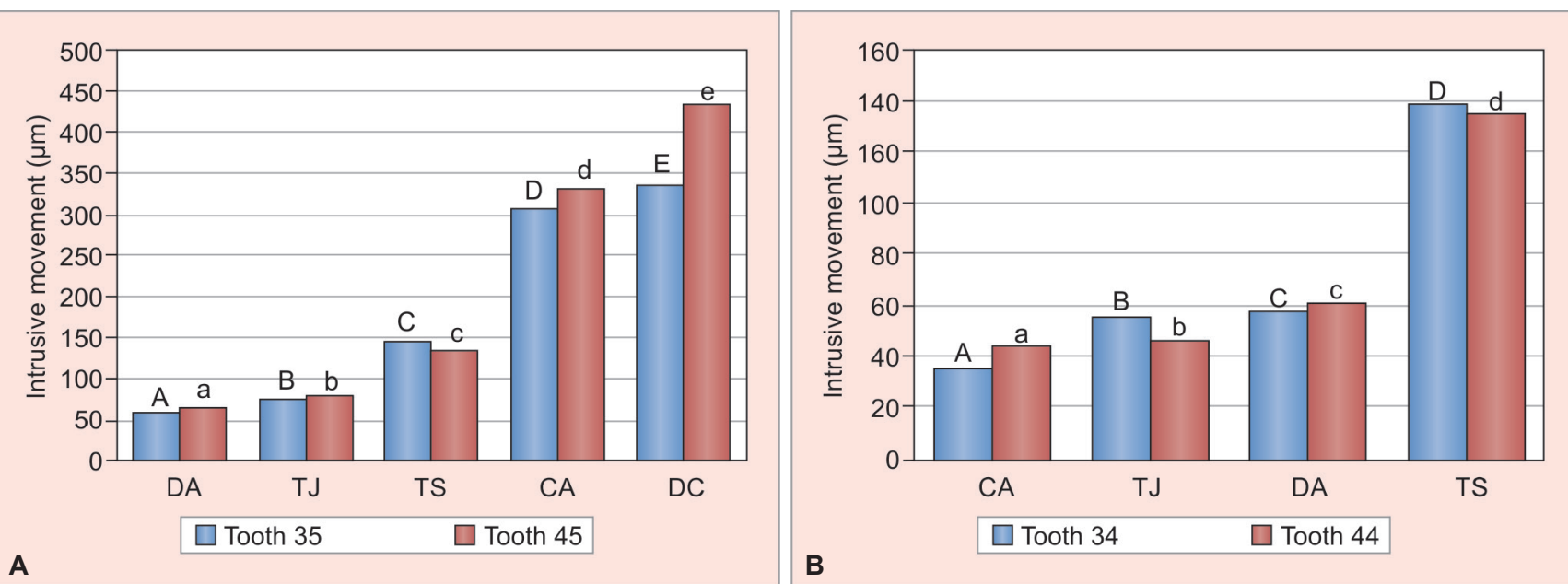

Graphs 1A and B: (A) Mean values of intrusive movement for teeth 35 and $45(\mu \mathrm{m})$ for the different treatment options are shown in an increasing order: TJ RPD; TS RPD; DA RPD; DC retained RPD; and (CA) cantilever bridge. Different capital letters denote a significant difference according to Tukey's pairwise comparison test for tooth 35 , while different small letters denote a significant difference according to Tukey's pairwise comparison test for tooth 45; and (B) Mean values of intrusive movement for teeth 34 and $44(\mu \mathrm{m})$ for the different treatment options in an increasing order: TJ RPD, TS RPD, DA RPD, and (CA) cantilever bridge. Different capital letters denote a significant difference according to Tukey's pairwise comparison test for tooth 34 , while different small letters denote a significant difference according to Tukey's pairwise comparison test for tooth 44
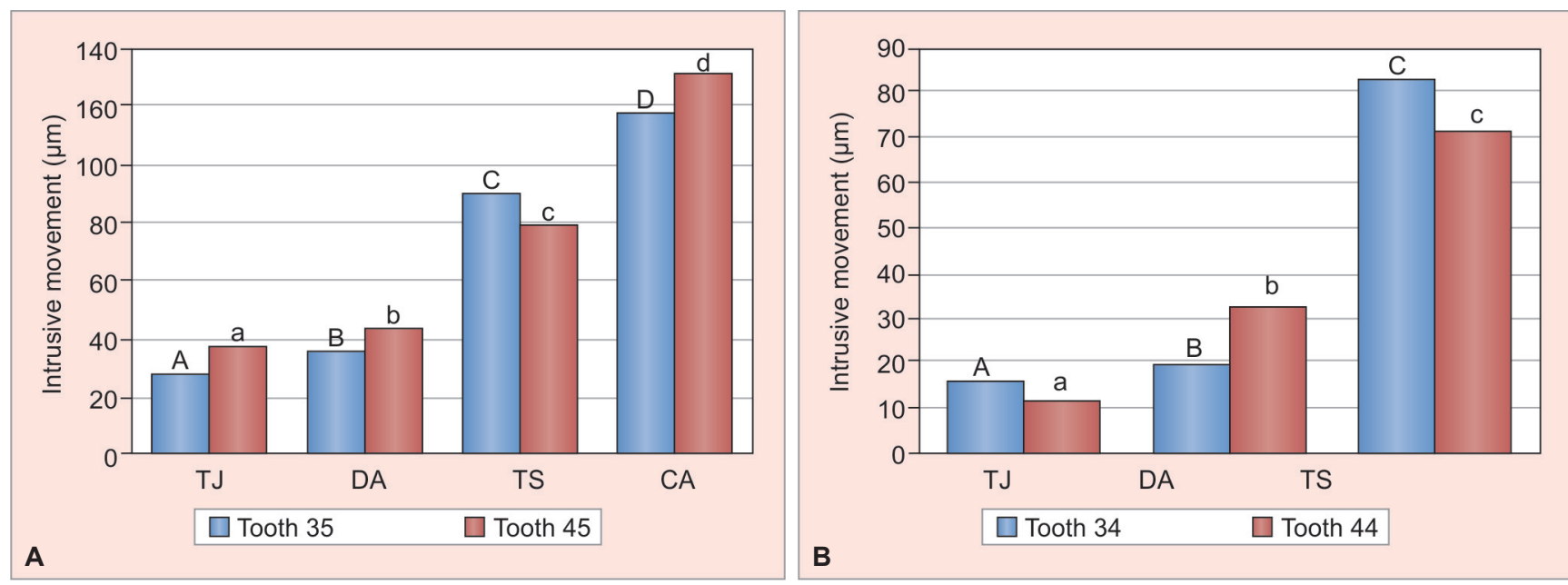

Graphs 2A and B: (A) Mean values of intrusive movement for teeth 35 and $45(\mu \mathrm{m})$ for the different treatment options in an increasing order: TJ RPD, TS RPD, DA RPD, and DC retained RPD. Different capital letters denote a significant difference according to Tukey's pairwise comparison for the significant ANOVA for tooth 35, while different small letters denote a significant difference according to Tukey's pairwise comparison test for tooth 45; and (B) Mean values of intrusive movement for teeth 34 and 44 ( $\mu \mathrm{m}$ ) for the different treatment options in an increasing order: TJ RPD; TS RPD; and DA RPD. Different capital letters denote a significant difference according to Tukey's pairwise comparison test for tooth 34, while different small letters denote a significant difference according to Tukey's pairwise comparison test for tooth 44

options in an increasing order: TJ RPD; TS RPD; and DA RPD. Different capital letters denote a significant difference according to Tukey's pairwise comparison test for tooth 34 , while different small letters denote a significant difference according to Tukey's pairwise comparison test for tooth 44 (Graph 2).

\section{Force applied onto the Tissue-supported Region of the Saddle (Position 3)}

The mean values of the intrusive movement in TS RPD, TJ RPD, DA RPD, and DC RPD in tooth 35 were 7.3, 8.1,
4.8, and $9.9 \mu \mathrm{m}$ respectively, and in tooth 45 were 7.5 , 8,5 , and $10.7 \mu \mathrm{m}$ respectively. The results of ANOVA revealed a statistically significant difference between the groups $(p<0.001)$. Bar chart shows mean values of intrusive movement for teeth 35 and $45(\mu \mathrm{m})$ for the different treatment options in an increasing order: TJ RPD, TS RPD, DA RPD, and DC-retained RPD. Different capital letters denote a significant difference according to Tukey's pairwise comparison test for tooth 35, while different small letters denote a significant difference according to Tukey's pairwise test for tooth 45 . The mean 


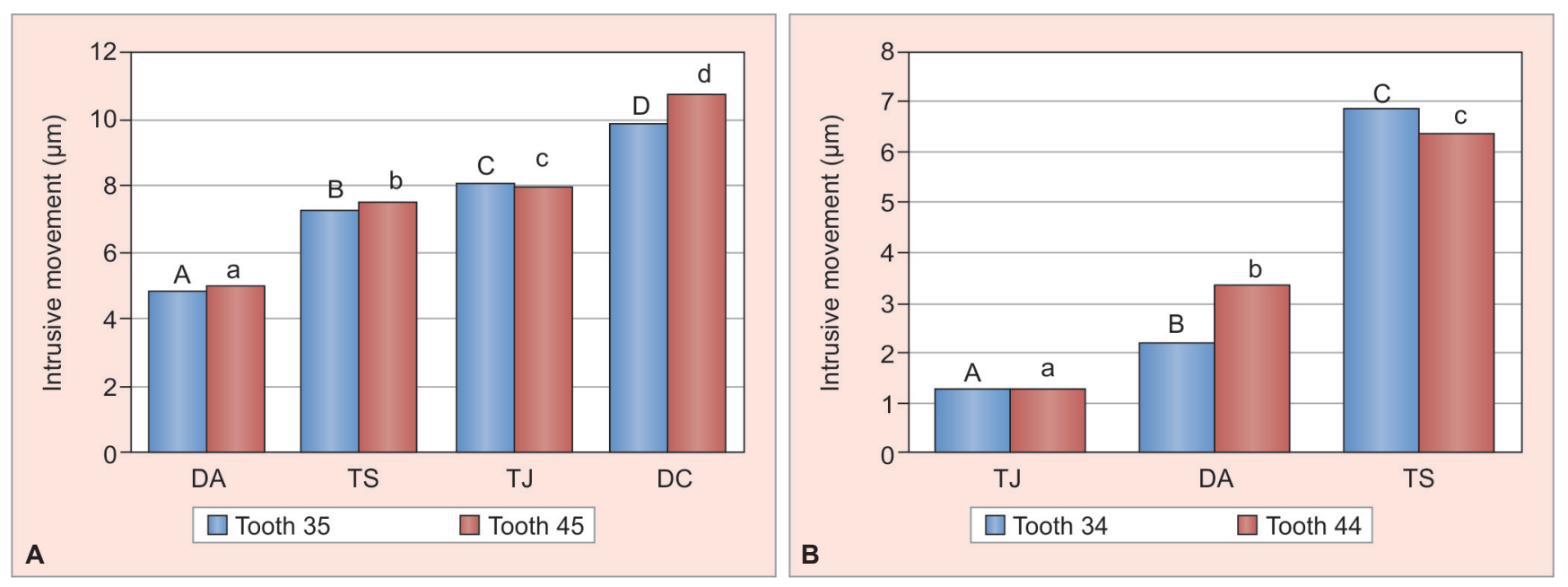

Graphs 3A and B: (A) Mean values of intrusive movement for teeth 35 and $45(\mu \mathrm{m})$ for the different treatment options in an increasing order: TJ RPD, TS RPD, DA RPD, and DC-retained RPD. Different capital letters denote a significant difference according to Tukey's pairwise comparison test for tooth 35, while different small letters denote a significant difference according to Tukey's pairwise test for tooth 45; and (B) Mean values of intrusive movement for teeth 34 and $44(\mu \mathrm{m})$ for the different treatment options in an increasing order: TJ RPD, TS RPD, and DA RPD. Different capital letters denote a significant difference according to Tukey's pairwise comparison test for tooth 34 , while different small letters denote a significant difference according to Tukey's pairwise comparison test for tooth 44

values of the intrusive movement in TS RPD, TJ RPD, and DA RPD in tooth 34 were $6.9,1.3$, and $2.2 \mu \mathrm{m}$ respectively, and in tooth 44 were $6.4,1.3$, and $3.4 \mu \mathrm{m}$ respectively. The results of ANOVA revealed a statistically significant difference between the groups $(p<0.001)$. Bar chart shows mean values of intrusive movement for teeth 34 and $44(\mu \mathrm{m})$ for the different treatment options in an increasing order: TJ RPD, TS RPD, and DA RPD. Different capital letters denote a significant difference according to Tukey's pairwise comparison test for tooth 34 , while different small letters denote a significant difference according to Tukey's pairwise comparison test for tooth 44 (Graph 3).

When loading on positions 2 and 3, the bending moment arm is longer than position 1 loading case. Thus, larger moment and, consequently, larger deflection ${ }^{8}$ is expected in positions 2 and 3 and less intrusion.

The cantilever bridge is tooth supported with no support from the residual ridge, so the abutment teeth are baring total load applied on the pontic. This is shown with the high intrusive movement on the second premolar $(35,45)$. Due to the leverage action of the cantilever bridge, the first premolars $(34,44)$ responded with extremely low intrusive movement.

The abutment nearest the pontic receives more than half the load placed against the pontic. ${ }^{9}$

Cantilevered pontic caused tilting and rotational movements without mentioning anything about the intrusive movements. ${ }^{10}$

The telescopic retained RPD, the intrusion of the $(35,45)$ abutments were low, and the values decreased gradually, with moving among positions 1,2 , and 3 .
The intrusion of the $(34,44)$ abutments was lower than $(35,45)$ as they are located away from the distal extension base, so lesser load transmitted, leading to less intrusive movement. The intrusion of the abutment teeth in TJ crowns was almost half of that of the TS crowns. The lower values of intrusion of abutments in TJ-retained RPD are due to the large surface area onto which load is applied.

Since stress is inversely proportional to area, as the area increases and the force applied is constant; the stress produced decreases. ${ }^{11}$

When the telescopic crowns were joined, this increased the surface area of the abutment teeth, leading to less stress, resulting in less intrusion of the abutment. ${ }^{12,13}$

In the DA (Bonwill)-retained RPD, the intrusion of the abutment teeth was low, and decreased gradually with moving from positions 1,2 , and 3 . This is due to distributing the stresses on both abutments of same side at the same time with the joined occlusal rests, and relative flexibility of the clasp. In addition, placing the occlusal rest on the occlusomesial surface of the second premolar decreases the fulcrum line and lever arm, leading to less movement of abutment teeth. In addition, disengagement of the clasps during loading decreases the stresses transmitted to the abutment teeth, so the residual ridge is bearing much of the load. ${ }^{14,15}$

In the DC-retained RPD, the intrusion of the abutment teeth was high and decreased gradually with moving from position 1, 2, and 3, as the residual ridge became more active in supporting and bearing the loads. Also by moving posteriorly, the forces transmitted became with a deflection effect rather than intrusive. In addition, 
the second premolars $(35,45)$ were the only used abutments, so they are the only abutments bearing the load, so more stress was transmitted to them, leading to more intrusion. Placing clasp on the occlusodistal surface of the abutment acts as a Class I lever leading to more intrusion of the abutment teeth. ${ }^{14}$ As already described, "this could be considered a cantilever design, and detrimental first-class lever force may be imparted to the abutment if tissue support under the extension base allows excessive vertical movement toward the residual ridge."

The DA clasp transmitted the least stresses causing movement of the abutment teeth in clasp-retained RPDs. ${ }^{6}$

The telescopic crowns caused less movement of the supporting teeth than cantilever bridge. ${ }^{13}$

The cantilever bridge and the DC-retained RPD caused the highest intrusive movement of the second premolars $(35,45)$ due to concentration of stresses on the second premolar. The separated telescope-retained partial denture followed them. Then, joined telescope and DA clasp-retained RPD caused the lowest intrusive movement of the abutment teeth.

The cantilever had the lowest intrusive movement on the first premolars $(34,44)$ due to the leverage action of the cantilever bridge. In the DC-retained RPD they were not utilized, so no forces of intrusive nature were transmitted to them. Then, the joined telescopic retained RPD and the DA clasp retained RPD. The separated telescopic-retained RPD caused the highest intrusive movement.

\section{CONCLUSION}

It can be concluded from this study that DA clasp with the joined telescopic crown-retained RPD produced the least intrusion of the abutment teeth; the DA claspretained RPD has the advantage of being cheaper than the telescopic-retained RPD. In addition, DA (Bonwill clasp) provides no need for crowning as in the joined telescopic-retained RPD.

\section{REFERENCES}

1. Abbas, NA. Clinical removable partial denture, 1st ed. Egypt: MSA University Press; 2008.

2. Abbas, NA. Removable partial denture technology, 2nd ed. Egypt: MSA University Press; 2010.

3. van Dalen A, Feilzer AJ, Kleverlaan CJ. A literature review of two-unit cantilevered FPDs. Int J Prosthodont 2004 MayJun;17(3):281-284.

4. Langer A. Telescope retainers for removable partial dentures. J Prosthet Dent 1981 Jan;45(1):37-43.

5. Eliason CM. RPA clasp design for distal-extension removable partial dentures. J Prosthet Dent 1983 Jan;49(1):25-27.

6. El-Wakad, MT. Evaluation of stresses and displacement in different clasp-retained partial dentures: in vitro study. Biomedical Engineering Department Faculty of Engineering. Helwan, Egypt: Helwan University; 2008.

7. Mizuuchi W, Yatabe M, Sato M, Nishiyama A, Ohyama T. The effects of loading locations and direct retainers on the movements of the abutment tooth and denture base of removable partial dentures. J Med Dent Sci 2002 Mar;49(1):11-18.

8. Aristizabal-Ochoa JD. Induced moments and lateral deflections in columns with initial imperfections and semi rigid connections: II. Verification and examples. Dyna (Bilbao) 2012;79:18-19.

9. Henderson D, Blevins WR, Wesley RC, Seward T. The cantilever type of posterior fixed partial dentures: a laboratory study. J Prosthet Dent 1970 Jul;24(1):47-67.

10. Himmel R, Pilo R, Assif D, Aviv I. The cantilever fixed partial denture-a literature review. J Prosthet Dent 1992 Apr;67(1):484-487.

11. Ambaum MH. General relationships between pressure, weight and mass of a hydrostatic fluid. Proc R Soc A 2008 Apr;464(2092):943-950.

12. Wenz HJ, Hertrampf K, Lehmann KM. Clinical longevity of removable partial dentures retained by telescopic crowns: outcome of the double crown with clearance fit. Int J Prosthodont 2001 May-Jun;14(3):207-213.

13. Shrestha P, Shrestha K. Telescopic crowns used as retainers for Kennedy class II removable partial dentures. J Nep Dent Assoc 2010;11:159-161.

14. Ben-Ur Z, Aviv I, Maharshak B. Factors affecting displacement of free-end saddle removable partial dentures. Quintessence Int 1991 Jan;22(1):23-27.

15. Carr, AB.; Brown, DT. McCracken's removable partial denture, 12th ed. USA: El Sevier Mosby; 2011. p. 105. 